\title{
Second Order Newton Iteration Method and Its Application to MOS Compact Modeling and Circuit Simulation
}

\author{
ZHIPING YU and ROBERT W. DUTTON \\ AEL 204, Center for Integrated Systems, Stanford University, Stanford, CA 94305, U.S.A.
}

\begin{abstract}
A robust second order Newton iteration scheme is proposed for solving the nonlinear algebraic equations. Potential applications include the projection of the initial guess to the solution when the parameters in the equations are changed. The mathematical derivation leading to the scheme is given and pros and cons of the method are discussed. As an example, the method has been applied to the evaluation of charge-sheet model for MOS as used in the circuit simulation.
\end{abstract}

Keywords: Numerical Algorithm, Newton Method, Nonlinear Equation, Second Order Iterative Scheme, MOS Modeling, Circuit Simulation

\section{INTRODUCTION}

Newton-Raphson method is one of the most effective methods in solving nonlinear equations. The success of this method largely lies on its linear nature, i.e., it uses the tangent line to approximate the behavior of the function, for which the zero-value solution is to be solved, around the current solution. It is therefore robust - straight line always has an intersect with the axis (of the coordinates) except when they are in parallel - as long as the initial guess falls in the convergence radius. And the convergence rate is quadratic once the iterative solution is close enough to the actual one. There has been incentive to improve the plain Newton method (or named first order Newton method for it uses the first order derivative only) for better convergence behavior and rate. The natural step would be to explore the second derivative, which represents the curvature of the function around the cur- rent solution and is sometimes not so difficult to evaluate. The problem with the straightforward extension of the Newton approach, i.e. retaining the power series up to the second degree in the Taylor expansion, is that the curve obtained to approximate the behavior of the function is a parabola which does not always have intersect with the axis and, even it has, there may be more than one intersect. So the efforts to seek higher order Newton iteration often introduce the instability to the method, not to mention the higher computational cost [1].

In this article, we will introduce a robust second order Newton method which retains the linear nature of the method, yet incorporates the information of the second order derivative in a natural way. The results of such an approach are the robustness and faster convergence rate. An additional benefit of using this higher order method is to have a better initial guess to the new solution when the parameters in the function change 
values. This application partially compensates the higher cost brought about by evaluating the second order derivatives for in the Newton iteration the foremost concern is to have a good initial guess to start with in order to guarantee the convergence. In this regard, the computation cost is of secondary concern.

In the next sections, the detailed mathematical process of deriving the second order Newton method and its projection extension are presented, followed by the application of the method to the charge-sheet MOS model [2]. Finally the conclusion is drawn.

\section{DERIVATION OF SECOND ORDER NEWTON ITERATION ALGORITHM}

The problem of solving a system of nonlinear (algebraic) equations is defined mathematically as follows:

$$
f(x, p)=0, \quad f, x, 0 \in \Re^{N}, p \in \Re^{M}
$$

where bold symbols represent vectors and $\mathrm{x}$ is the basic variable(s) such as the surface potentials in the charge-sheet model and $\boldsymbol{p}$ is the parameter(s) such as the applied bias. Assuming for now $M=N=1$, the conventional Newton-Raphson method updates the solution as follows

$$
x^{i+1}=x^{i}+\Delta x^{i}, \quad \Delta x^{i}=-f /\left.f_{x}\right|_{x=x^{i}}
$$

which is obtained from the Taylor expansion of function $f(x)$ at $x=x^{i}$ and retaining only the first power term of $\Delta x$. That is,

$$
f(x+\Delta x)=f(x)+f_{x}(x) \Delta x
$$

Now we try to derive a second order Newton iteration scheme by retaining higher degree terms, i.e. second power term, as follows:

$$
f(x+\Delta x)=f(x)+f_{x}(x) \Delta x+\frac{1}{2} f_{x x}(x) \Delta^{2} x
$$

Let $f(x+\Delta x)=0$ and solve for $\Delta x$, one would obtain the following solutions

$$
\Delta x=\frac{-f_{x} \pm \sqrt{f_{x}^{2}-2 f_{x x} f}}{f_{x x}}
$$

This formulation for the correction, $\Delta x$, cannot directly be used in Eq. (2) (first part) for two reasons: (1) there are two solutions and the values may end up imaginary if the argument of the square root is negative; (2) when the second derivative, i.e. $f_{x x}$, is zero, the solution is singular. However, if the square root term is expanded in Taylor series and up to the second power terms are retained, i.e.,

$$
\sqrt{1+x}=1+\frac{1}{2} x-\frac{1}{8} x^{2}
$$

for $x \ll 1$, Eq. (5) can be approximated to

$$
\Delta x=-\frac{f}{f_{x}}\left(1+\frac{1}{2} \frac{f}{f_{x}^{2}} f_{x x}\right)
$$

where in the approximation process we only retains the smallest solution where $f_{x}$ in the numerator in Eq. (5) is canceled out among two possible solutions. Expression (7) has several nice features: (1) It retains the linear nature of the first order Newton method; (2) The second order derivative represents only a correction to the original slope $\left(f_{x}\right)$ of the tangent line and if $f_{x x} \rightarrow 0$, Eq. (7) is simply reduced to Eq. (2) (right part). It is Eq. (7) that is called the second order Newton iteration scheme.

\section{EXTENSION TO THE INITIAL GUESS PROJECTION}

As has been discussed in [3], the Newton method can be used to project the initial guess to the new solution when the parameters in the function are changed. With the scheme of the second order Newton iteration (Eq. 7), the same methodology can be applied to obtain a formula for the initial guess projection. The process of derivation is briefly summarized as follows.

$$
\begin{array}{r}
f(x+\Delta x, p+\Delta p)=f(x, p)+f_{x} \Delta x+\frac{1}{2} f_{x x} \Delta^{2} x+f_{p} \Delta p \\
+\frac{1}{2} f_{p p} \Delta^{2} p+f_{x p} \Delta p \Delta x
\end{array}
$$

The goal here is to find an expression for $\Delta x$ such that $f(x+\Delta x, p+\Delta p)=0$ for a given $\Delta p$. The result after certain approximations are made is

$$
\Delta x=-\frac{f_{p} \Delta p+\frac{1}{2} f_{p p} \Delta^{2} p}{f_{x}+f_{x p} \Delta p}\left[1+\frac{f_{p} \Delta p+\frac{1}{2} f_{p p} \Delta^{2} p}{\left(f_{x}+f_{x p} \Delta p\right)^{2}} f_{x x}\right]
$$


which, when all the second derivative terms become zero, is reduced to

$$
\Delta x=-\frac{f_{p}}{f_{x}} \Delta p
$$

In the conventional Newton iteration process of finding the solution $x, f_{x}$, or called Jacobian matrix for multi-dimensional cases, is already known and $f_{p}$ is usually easy to compute, so the evaluation of Eq. (10) is not computation intensive. The evaluation of Eq. (9) needs much more effort and can only be justified when an improved initial guess is absolutely necessary. For detailed discussion of applications for the Newton projection, refer to [3].

\section{APPLICATIONS}

In principle, the above schemes -- Newton iteration, Eq. (7), and projection, Eq. (9) -- can be applied to any nonlinear equation solver. However, because of the complexity of evaluating the second derivatives it is only practical when the dimension of vector $\boldsymbol{x}$ in Eq. (1) is small. Note that when $N>1, f_{x}$ represents an $N \times N$ matrix (the Jacobian matrix) while $f_{x x}$ represents an $N \times N \times N$ tensor, which means $N$ times increase in both size and computational effort. So it is more practical to apply this higher order iteration scheme to the circuit simulation where only compact models, closed form or not, are used than in more detailed technology simulations such as the device simulation. In the following, we apply the second order Newton iteration scheme together with its projection counterpart to the charge-sheet MOS model [2].

The advantage of the charge-sheet MOS model over the more popular analytical, compact MOS model such as MOS level 1-3 used in SPICE is that it does not partition the MOS operation region into several sub-regions such as linear vs. saturation regions and weak (sub-threshold) vs. strong inversion regions. So the higher order derivatives of basic $I-V$ (and $Q-V$ ) characteristics such as conductances and capacitances are guaranteed to be continuous. In addition, the charge-sheet model naturally includes both the diffusion and drift current components in the channel conduction. So this model is particularly suitable to the analog and low-power circuit applications. Even though this model was first proposed in 70 [2], it has recently drawn much attention in the compact circuit device modeling, e.g. see [4]. The problem, however, related to the charge-sheet model as compared to other compact MOS models is that it cannot be expressed as a closed form. Numerical iterations have to be used to obtain the device terminal response to the bias change. The computational efficiency thus becomes a major concern in applying this model to the circuit simulation. Right in this respect, the second order Newton method can play an active role. Even though the tests done are still preliminary as this article is written, the results shown below indicate the promising nature of the method in this particular application.

In the charge-sheet model, two basic quantities have to be first determined before the terminal currents (or stored charges associated with the terminals) can be calculated. These two quantities are the surface potentials at the source $\left(\psi_{S 0}\right)$ and drain $\left(\psi_{S L}\right)$ ends of the channel and are dependent upon the gate-source $\left(V_{G S}\right)$ and drain-source $\left(V_{D S}\right)$ biases through transcendental equations. Given bias, the surface potentials have to be solved iteratively.

We first examine the solution of $\psi_{S 0}$ which depends on $V_{G S}$ only via the following equation:

$$
\begin{aligned}
C_{0 x}\left(V_{G S}-V_{f b}-\Psi_{S 0}\right) & =q N_{A} L_{D} \sqrt{2} \\
& {\left[\frac{\Psi_{S 0}}{V_{T}}-1+\left(\frac{n_{i}}{N_{A}}\right)^{2} e^{\Psi_{S 0} / V_{T}}\right]^{1 / 2} }
\end{aligned}
$$

where $L_{D}$ is the Debye length and all other symbols have the conventional meanings. For the origin of this equation, refer to [2]. Figure 1 and Table I show the comparison of the above solution for $V_{G S}=1.3 \mathrm{~V}$ using both conventional (i.e. 1st order) and second order Newton iteration methods. Apparently, for the same accuracy, the second Newton method cuts the iteration times by one half compared to its 1 st order sibling. Also shown in Figure 1 is the curve (a parabola) for the complete second order Newton approach as represented in Eq. (4). There are two solutions for 


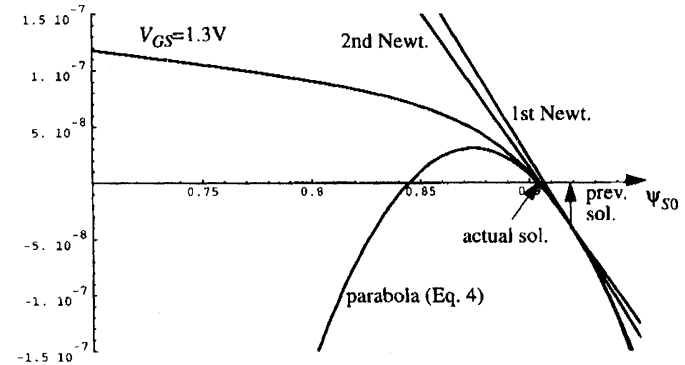

FIGURE 1 Solution process for Eq. (11) showing the merit of different schemes. The 2 nd Newton apparently prevails

TABLE I Iterations for solving $\psi S_{0}$ when $V_{G S}$ is set to $1.3 \mathrm{~V}$

\begin{tabular}{lcc}
\hline Iteration \# & 2nd Newton & 1st Newton \\
\hline 0 (projected) & 0.836712 & 0.877432 \\
1st & 0.768428 & 0.830788 \\
2nd & 0.767125 & 0.783837 \\
3rd & & 0.767611 \\
4th & & 0.767126 \\
\hline
\end{tabular}

the updates and one is far from the actual solution. However, the proposed 2nd Newton method is represented by a straight line as is in the 1st order Newton method but the slope is modified by the curvature of the original function from the mere tangent. It is clear that this slope modification brings the intersect of the sector (i.e. line) with the $x$-axis more close to the final solution than that from the tangent line.

We then demonstrate how the projection scheme spawned from this 2 nd order Newton method works in the charge-sheet model. The equation for determining $\psi_{S L}$ given $V_{D S}$ and knowing $\psi_{S 0}$ is as follows [2]:

$$
\begin{aligned}
\beta V_{D S} & =\beta\left(\Psi_{S L}-\Psi_{S 0}\right)-\ln \left(1-\frac{\left.C_{0 x} \Psi_{S L}-\Psi_{S 0}\right)}{q N(0)}\right. \\
- & \left.\frac{q N_{A} L_{D} \sqrt{2}}{q N(0)}\left[\sqrt{\beta \Psi_{S L}-1}-\sqrt{\beta \Psi_{S 0}-1}\right]\right)
\end{aligned}
$$

where $\beta=1 / V_{T}$. We now consider $V_{D S}$ as the parameter to see how its change will affect the solution of $\psi_{S L}$. The results are shown in Figure 2 and Table II. Essentially, once the solution, i.e. $\psi_{S L}$, is obtained for

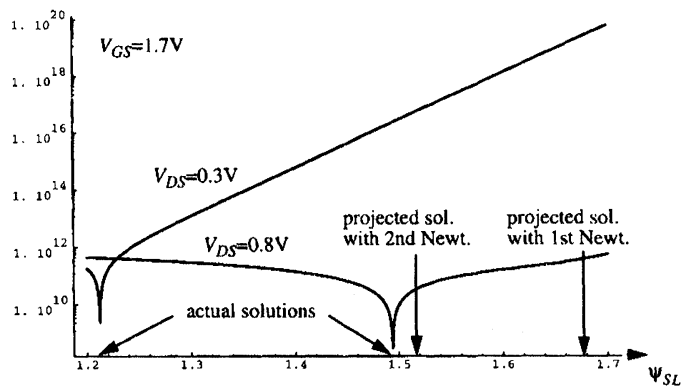

FIGURE 2 Solution process for $\psi_{S L}$ at $V D S=0.8 \mathrm{~V}$ based on the solution of $V D S=0.3 \mathrm{~V}$. The 2nd Newton gives superior initial guess. Due to the log scale the function is plotted in absolute values

Iterations for solving $\psi_{S L}$ when $V_{D S}$ is changed from 0.3 to $0.8 \mathrm{~V}$ for fixed $V_{G S}=1.7 \mathrm{~V}$

\begin{tabular}{lcc}
\hline Iteration \# & 2nd Newton & 1st Newton \\
\hline 0 (projected) & 1.50961 & 1.66983 \\
$1 \mathrm{st}$ & 1.49407 & 1.59622 \\
2nd & 1.494075989737123 & 1.50233 \\
3rd & & 1.49408 \\
4 th & 1.494075989737396 \\
5 th & 1.494075989737122 \\
\hline
\end{tabular}

order projection) is almost as good as the final solution whereas the projection from the 1 st order method, though still leading to the convergence of the iteration, is much far away from the final solution as a comparison. Of course, those improvements in the projection and cut in the iteration times are achieved at the expense of evaluating the second order derivatives. This higher order scheme has also found application in the fast circuit simulation [5] to predict the region crossing.

\section{CONCLUSIONS}

The information on the second order derivatives of the function (or equivalently, the curvature of the 
function) can be explored to improve the conventional Newton iteration in both convergence behavior and efficiency while retaining the robust nature of the method. The actual saving (or penalty) of the computation effort depends on the nature of the problem to be solved and asks for further investigation and numerical experiments. One thing is certain, however, that in the tough to-converge problems this higher order method provides an precious alternative for the solution-finding because except of the uncertainty in the computation cost, this method is guaranteed to perform better than the conventional Newton method.

\section{References}

[1] J.M. Ortega and W.C. Rheinboldt, Iterative solution of nonlinear equations in several variables, New York, Academic Press, 1970.

[2] J.R. Brews, "A charge-sheet model of the MOSFET", SolidState Elec, Vol. 21, p. 345, 1978.

[3] Z. Yu, R.W. Dutton, and M. Vanzi, "An extension to Newton method in device simulators on an efficient algorithm to evaluate small signal parameters and to predict initial guess," IEEE Trans. CAD, vol. CAD-6, no.1, p.41, Jan. 1987.

[4] M. Miura-Mattausch, U. Feldmann, A. Rahm, M. Bollu, and D. Savignac, "Unified complete MOSFET model for analysis of digital and analog circuits," Digest ICCAD,p. 264, 1994.

[5] Y-H. Shih and S-M. Kang, "Analytic transient solution of general MOS circuit primitives," IEEE Trans. CAD, vol. 11, no. 6, p. 719, June 1992.

\section{Biographies}

Zhiping Yu is a Senior Research Associate at Dept. of Electrical Engineering in Stanford University. His research interests include device and circuit simulation, numerical algorithm development and MOS compact modeling.

Robert W. Dutton is a Professor of Electrical Engineering at Stanford University and an IEEE Fellow. His research interests include IC process and device simulation, high performance computing, and application of electrical disciplines to the environmental protection. 

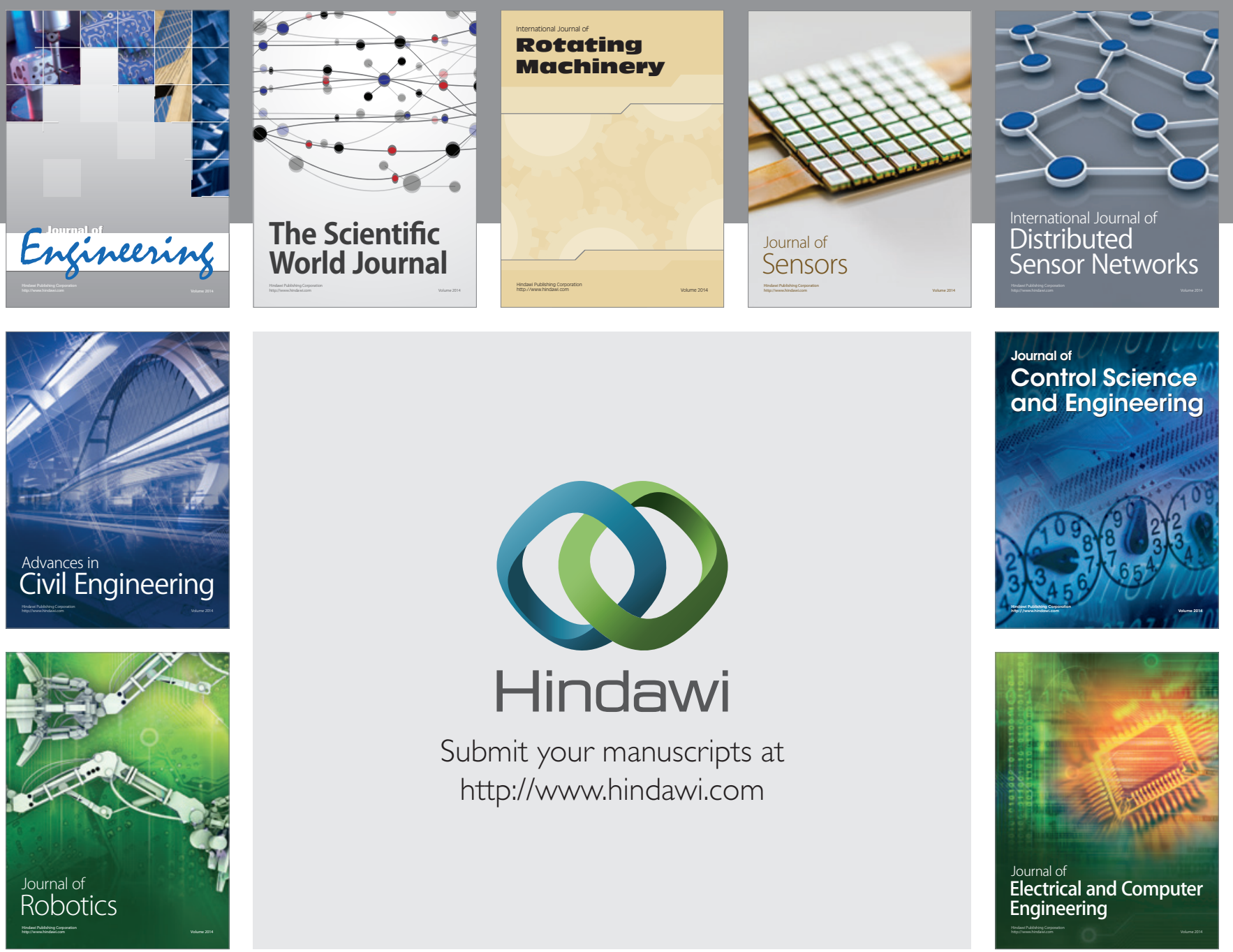

Submit your manuscripts at

http://www.hindawi.com
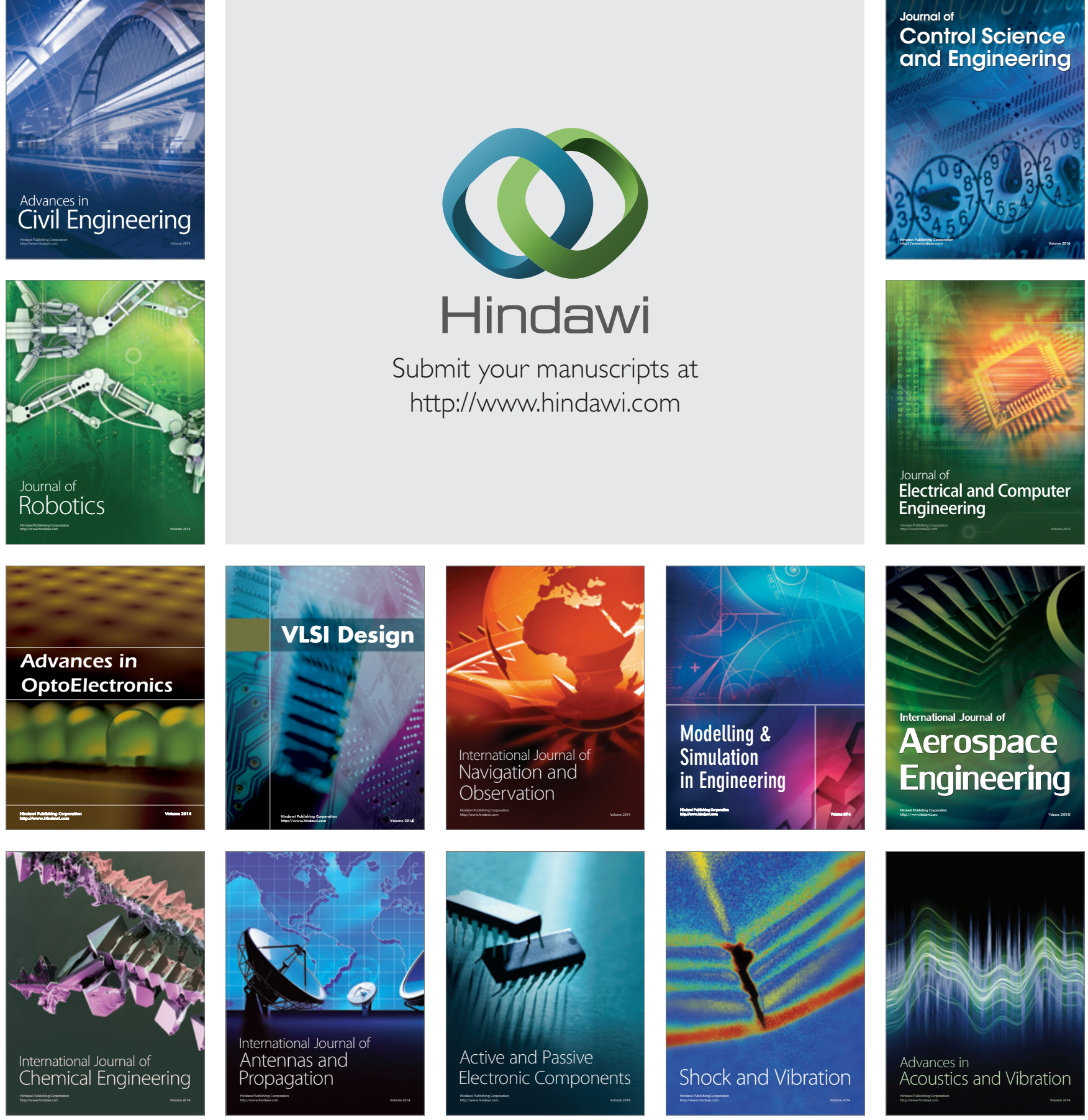